\title{
QUANTIFICAÇÃO DE MARTENSITA EM AÇO INOXIDÁVEL POR ENSAIO DE CORRENTES PARASITAS*
}

\author{
Vitor Manoel de Araújo Silva ${ }^{1}$ \\ Cesar Giron Camerini \\ Juan Manuel Parda/ ${ }^{3}$ \\ Juan Carlos Garcia de Blás ${ }^{4}$ \\ Gabriela Ribeiro Pereira ${ }^{5}$
}

\section{Resumo}

Os aços inoxidáveis austeníticos são materiais utilizadas nos ramos petroquímico, nuclear e aeroespacial cujas demandas se resumem essencialmente à: boa resistência mecânica, excelente soldabilidade e elevada resistência à corrosão. Apesar destes metais atenderem a estes requisitos, não raro são submetidos a etapas de conformação para elaboração de peças, o que pode modificar as suas propriedades originais. Basicamente, a causa desta mudança são alterações metalúrgicas induzidas por deformação, como a transformação da austenita (paramagnética) em martensita-a' (ferromagnética). Tendo em vista que tais mudanças são acompanhadas de variações nas propriedades elétricas/ magnéticas destes aços, uma maneira de monitorá-los de maneira não destrutiva é através da técnica de correntes parasitas. Para este estudo, diversas amostras de aço inoxidável AISI 321 foram solubilizadas e deformadas por laminação a fim de produzirem diferentes frações volumétricas de martensita. Em seguida, submetidas à microscopia ótica, magnetômetro de amostra vibrante e correntes parasitas. De acordo com os resultados, percebe-se que toda a transformação martensítica se passa no intervalo entre 0,10 e 0,55 de deformação verdadeira. Além disso, com os dados de magnetização de saturação, foi possível estabelecer uma curva de calibração de comportamento exponencial, permitindo a quantificação da martensita de maneira não destrutiva.

Palavras-chave: Aço inoxidável austenítico; Deformação a frio; Transformação martensítica; Correntes parasitas.

\section{STAINLESS STEEL MARTENSITE QUANTIFICATION BY MEANS OF EDDY CURRENT TESTING}

\section{Abstract}

Austenitic stainless steels are materials in petrochemical, nuclear and aerospace industries whose demands are essentially summarized as: good mechanical strength, excellent soldability and high resistance to corrosion. Although these metals meet these requirements, the conformation steps for the preparation of parts, which can modify as their original properties, are common. Basically, a cause of this change is a transformation of austenite (paramagnetic) into martensite- $\alpha^{\prime}$ (ferromagnetic). Considering that such changes are accompanied by variations in the electrical / magnetic properties of these steels, a way of non-destructively monitoring them is through the eddy current testing. For this study, several samples of AISI 321 stainless steel were solubilized and deformed by cold rolling in order to produce different martensite volumetric fractions. Then, subjected to optical microscopy, vibrating sample magnetometer and eddy current testing. According to the results, the whole martensitic transformation occurs between 0.10 and 0.55 of true strain. In addition, with the saturation magnetization data, it was possible to establish a calibration curve of exponential behavior, allowing non-destructive martensite quantification.

Keywords: Austenitic stainless steel; Cold rolling; Martensitic transformation; Eddy current.

1 Engenharia de Materiais, M.Sc., Doutorando, Programa de Engenharia Metalúrgica e de Materiais, COPPE/UFRJ, Rio de Janeiro, RJ, Brasil. 
2 Engenharia de Controle e Automação, M.Sc., Pesquisador, LNDC, COPPE/ UFRJ, Rio de Janeiro, RJ, Brasil.

3 Engenharia Mecânica, D.Sc, Professor, Programa de Engenharia Mecânica, UFF, Niterói, RJ, Brasil.

4 Engenharia Metalúrgica, D.Sc., Professor, Programa de Engenharia Metalúrgica e de Materiais, COPPE/UFRJ, Rio de Janeiro, RJ, Brasil.

5 Engenharia Nuclear, D.Sc., Professora, Programa de Engenharia Metalúrgica e de Materiais, COPPE/UFRJ, Rio de Janeiro, RJ, Brasil. 


\section{INTRODUÇÃO}

Os aços inoxidáveis austeníticos (AIA) vêm sendo amplamente estudados por conta da transformação de austenita em martensita- $\alpha^{\prime}$ induzida por deformação [1-3]. A facilidade desta transformação está diretamente ligada à energia de falha de empilhamento (EFE), a qual depende da composição química, temperatura e taxa de deformação [4]. O processo de formação da martensita- $\alpha^{\prime}$ inicia-se com a transformação da austenita $(\mathrm{\gamma})$ em martensita- $\varepsilon$ e, finalmente, em $\alpha$ '. Para entender como a martensita- $\varepsilon$ se forma, é valido destacar que em uma estrutura CFC, como na austenita, os planos da família $\{111\}$ podem ser representados como uma sequência de planos "ABCABCABC". Porém, por conta da deformação plástica, uma discordância integral pode dissociar-se em duas discordâncias de Shockley, gerando uma falha de empilhamento naquela região. Neste caso, o empilhamento torna-se uma sucessão de planos da forma "ABABAB", equivalente aos planos $\{0001\}$ da estrutura $\mathrm{HC}$, a mesma da martensita- $\varepsilon$. Assim, quanto menor a EFE mais fácil a decomposição da discordância e, consequentemente, a formação de martensita- $\varepsilon$ e $\alpha^{\prime}[4]$.

Para detectar e quantificar a martensita-a', diversos pesquisadores vêm empregando métodos como: magnetômetro de amostra vibrante (VSM) [5], ferritoscópio [6], microscopia eletrônica de varredura [7], difração de raios X (DRX) [8], entre outros. Porém, poucos são os estudos que trazem o método de correntes parasitas (ECT) como forma de identificar e quantificar a martensita. Em um deles [9] algumas amostras dos aços AISI 321 e 304 foram reduzidas na sua espessura entre $20 \%$ e 60 $\%$ e inspecionadas com uma sonda absoluta em uma única frequência de operação. Através da observação do plano de impedância, foi possível separar cada um dos estados de deformação, indicando a formação de uma fase ferromagnética (martensita-a'). Em outro estudo, um pouco mais aprofundado [10], peças de aço AISI $304 \mathrm{~L}$ foram deformadas entre $10 \%$ e $50 \%$ em suas espessuras e investigadas com frequências de operação entre $0,05 \mathrm{kHz}$ e $10 \mathrm{kHz}$. Os resultados identificaram mudanças no comportamento eletromagnético da liga na faixa de deformações e, através da amplitude do sinal, foi estabelecida uma relação exponencial entre a amplitude e a fração volumétrica de martensita- $\alpha^{\prime}$.

O objetivo deste trabalho é estabelecer uma calibração para o equipamento de correntes parasitas, a fim de permitir medidas da fração volumétrica de martensita- $\alpha^{\prime}$ de forma não destrutiva. 


\section{MATERIAIS E MÉTODOS}

Nove amostras de aço AISI 321 com dimensões de $80 \times 70 \times 9,5 \mathrm{~mm}^{3}$ foram cortadas de uma chapa com a composição química mostrada na Tabela 1. As amostras foram retiradas com seus comprimentos na mesma direção de laminação da chapa. Em seguida, solubilizadas durante 30 minutos a $1100^{\circ} \mathrm{C}$.

Tabela 1. Composição química do aço AISI 321 (\% peso) - balanço de Fe

\begin{tabular}{|l|l|l|l|l|l|l|l|l|l|l|l|}
\hline $\mathrm{C}$ & $\mathrm{Mn}$ & $\mathrm{Si}$ & $\mathrm{P}$ & $\mathrm{S}$ & $\mathrm{Cr}$ & $\mathrm{Ni}$ & $\mathrm{Mo}$ & $\mathrm{Al}$ & $\mathrm{Cu}$ & $\mathrm{N}$ & $\mathrm{Ti}$ \\
\hline 0,009 & 0,82 & 0,48 & 0,036 & 0,002 & 17,05 & 9,04 & 0,038 & 0,006 & 0,054 & 0,009 & 0,122 \\
\hline
\end{tabular}

O processo de deformação foi realizado em um laminador duo reversível ao longo de diversos passes de laminação, sendo a redução média ao redor de 0,1 $\mathrm{mm}$ por passe. Entre cada passe as amostras foram resfriadas em água à temperatura ambiente $\left(25^{\circ} \mathrm{C}\right)$ e a espessura medida com um paquímetro em três pontos diferentes. As deformações verdadeiras $(\mathrm{dv})$ impostas e a espessura final $\left(\mathrm{e}_{\mathrm{f}}\right)$ de cada amostra podem ser verificadas na Tabela 2. Após a laminação, amostras para a microscopia ótica foram cortadas e preparadas com lixamento, polimento e ataque. Nesta última etapa, foi realizado um ataque eletrolítico com ácido nitrico $60 \%$ e um ataque com ferrofluido visando a revelar a martensita- $\alpha$ '.

Tabela 2. Características das amostras produzidas através da deformação a frio

\begin{tabular}{|c|c|c|}
\hline Amostra & Deformação verdadeira (dv) & ef $(\mathrm{mm})$ \\
\hline AM -1 & 0,00 & 9,5 \\
\hline AM - & 0,05 & 9,0 \\
\hline AM - 3 & 0,10 & 8,7 \\
\hline AM - 4 & 0,15 & 8,2 \\
\hline AM - 5 & 0,20 & 7,8 \\
\hline AM - 6 & 0,27 & 7,3 \\
\hline AM - 7 & 0,40 & 6,4 \\
\hline AM - 8 & 0,55 & 5,4 \\
\hline AM - -9 & 1,00 & 3,5 \\
\hline AM - 10 & 1,98 & 1,3 \\
\hline AM - 11 & 2,47 & 0,8 \\
\hline AM - 12 & 2,94 & 0,5 \\
\hline
\end{tabular}


Para estimar a fração volumétrica de martensita, foi utilizado os valores de magnetização de saturação de cada amostra $\left(m_{s}\right)$ e de magnetização de saturação intrínseca da martensita $\left(\mathrm{m}_{\mathrm{si}}\right)$, todos estes obtidos no VSM. Neste equipamento, foram utilizadas amostras cilíndricas de 2,6 $\mathrm{mm}$ de altura por 3,0 $\mathrm{mm}$ de diâmetro, cortadas por eletroerosão. A partir dos resultados do VSM e de acordo com estudo anterior [11] a fração de martensita- $\alpha^{\prime}\left(\mathrm{C}_{\alpha^{\prime}}\right)$ pôde ser obtida de acordo com a Equação 1.

$$
C_{\alpha \prime}=\frac{m_{s}}{m_{s i}}
$$

Para o método de correntes parasitas, foi utilizado o equipamento OmniScan MX ECA com uma sonda absoluta operando a uma única frequência. Uma calibração com um bloco padrão foi também realizada a fim de estabelecer, no plano de impedância elétrica, claras diferenças entre o comportamento magnético e não-magnético da liga. Neste processo, materiais com comportamento magnético foram colocados com ângulos de fase variando entre $0^{\circ}$ e 90․ Já os materiais não-magnéticos, com respostas entre $0^{\circ}$ e $-90^{\circ}$. Os parâmetros utilizados na calibração podem ser verificados na Tabela 3.

Tabela 3. Parâmetros utilizados para calibração no equipamento de correntes parasitas

\begin{tabular}{|l|l|}
\hline Frequência de operação & $8 \mathrm{kHz}$ \\
\hline Alimentação & $4 \mathrm{~V}$ \\
\hline Ganho & $37 \mathrm{~dB}$ \\
\hline Rotação & $270^{\circ}$ \\
\hline
\end{tabular}

\section{RESULTADOS E DISCUSSÃO}

Na Figura 1 é possível observar ripas de martensita bem como grãos austeníticos. A martensita pode ser observada como os locais mais escuros, assim como reportou [6], no interior dos grãos austeníticos. A formação da martensita nesta amostra não era esperada uma vez que amostra não foi submetida à laminação. Sua formação, porém, pode ser explicada pelo lixamento superficial realizado para a micrografia, que introduziu energia suficiente para a formação de $\alpha$ '. Estes resultados são semelhantes aos encontrados em [12]. A Figura 2 revela a amostra deformada à $0,55 \mathrm{dv}$, onde é possível observar os grãos austeníticos alongados na direção da laminação. Observase também a presença das regiões escuras que equivalem à martensita. Além disso, como indicado na imagem e identificado em trabalho anterior [12] nota-se a presença de nitreto de titânio (TiN), na imagem. 


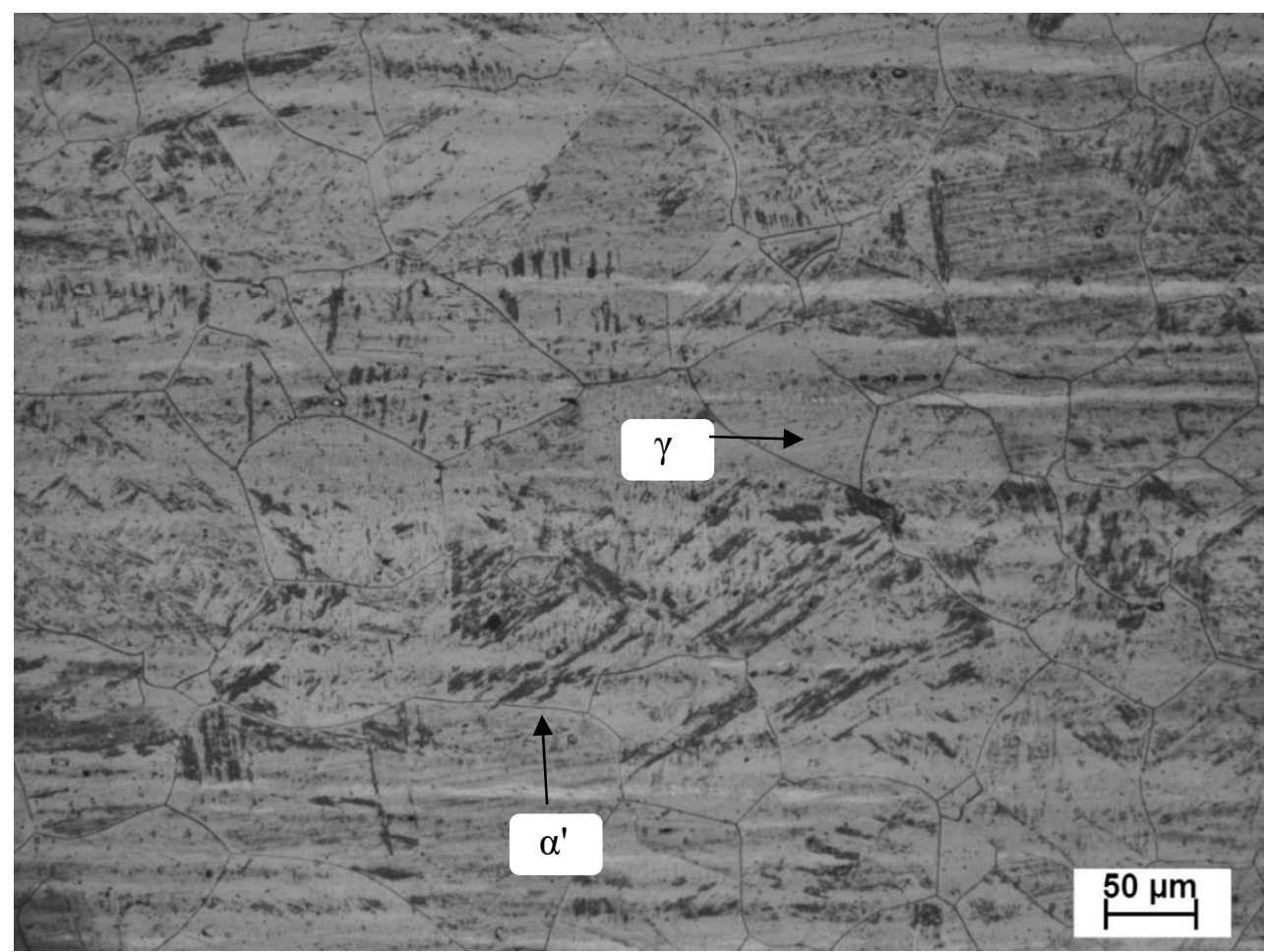

Figura 1. Amostra não deformada. Regiões mais escuras representam martensita $\alpha^{\prime}$.

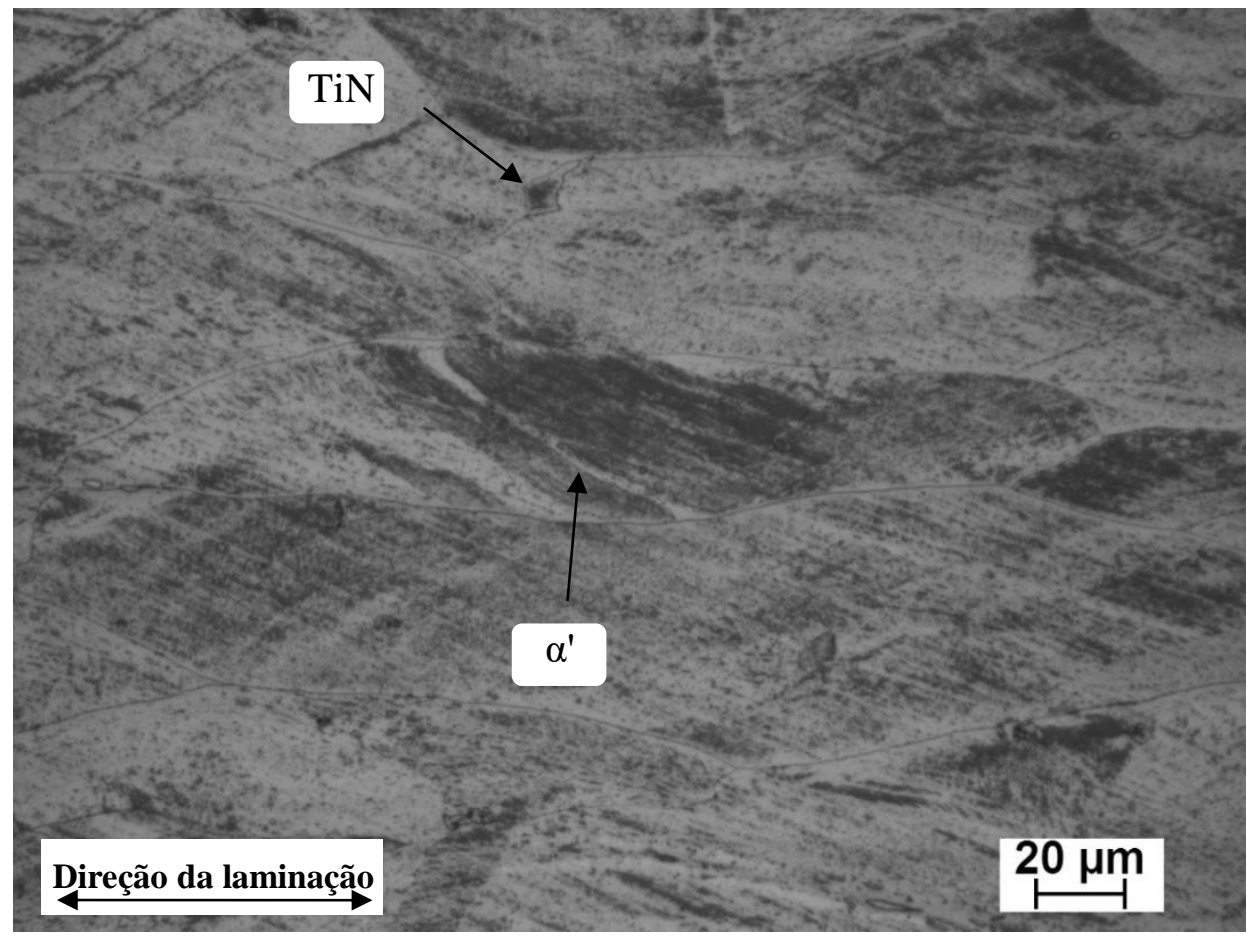

Figura 2. Microscopia ótica para a amostra deformada a 0,55 dv. Destaca-se a formação de martensita $\alpha$ ', além dos grãos alongados. 
Os resultados de magnetização de saturação obtidos pelo VSM e transformados em fração volumétrica de martensita pela Equação 1, podem ser observados na Figura 3. Nela, nota-se que a transformação martensítica aumenta com a deformação, iniciando-se ao redor de 0,10 dv e chegando ao seu máximo em torno de 0,50 dv. A curva tem um formato sigmoidal e resultados semelhantes podem ser encontrados na literatura [8-10]. A fração de martensita obtida pelo VSM foi escolhida em detrimento de outros métodos de quantificação, como por exemplo a difração de raios $X$, pois este último é muito influenciado pelas condições da superfície, como lixamento e polimento, além da textura [13]. Outrossim, de acordo com [14] a quantidade de martensita induzida por deformação é maior na superfície do que no restante da amostra. Logo, como as amostras para o VSM são cilíndricas e retiradas do interior das amostras, a influência da superfície é mitigada e maior quantidade de material é analisa, levando a resultados mais confiáveis.

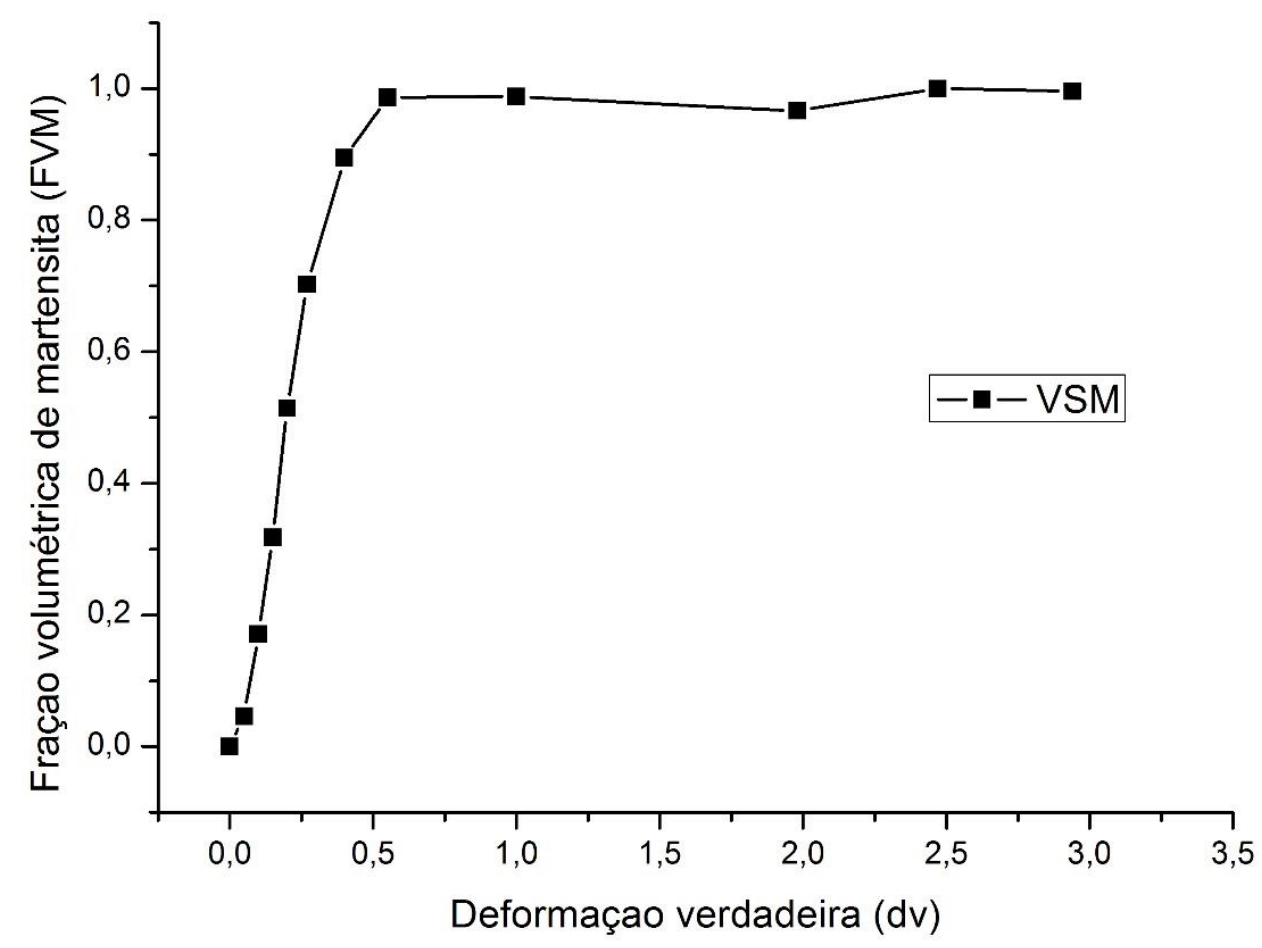

Figura 3. Fração volumétrica de martensita obtida pelo VSM.

A partir dos resultados de quantificação da fração volumétrica de martensita (FVM) anteriores e com os dados do ângulo de fase (AF), foi possível estabelecer uma calibração, a fim de servir de base para a determinação da fração de martensita diretamente a partir do ensaio de correntes parasitas. A melhor curva obtida foi a de comportamento exponencial (coeficiente de correlação $=0,99$ ), mostrada na Figura 4 , e que segue a Equação 2. 


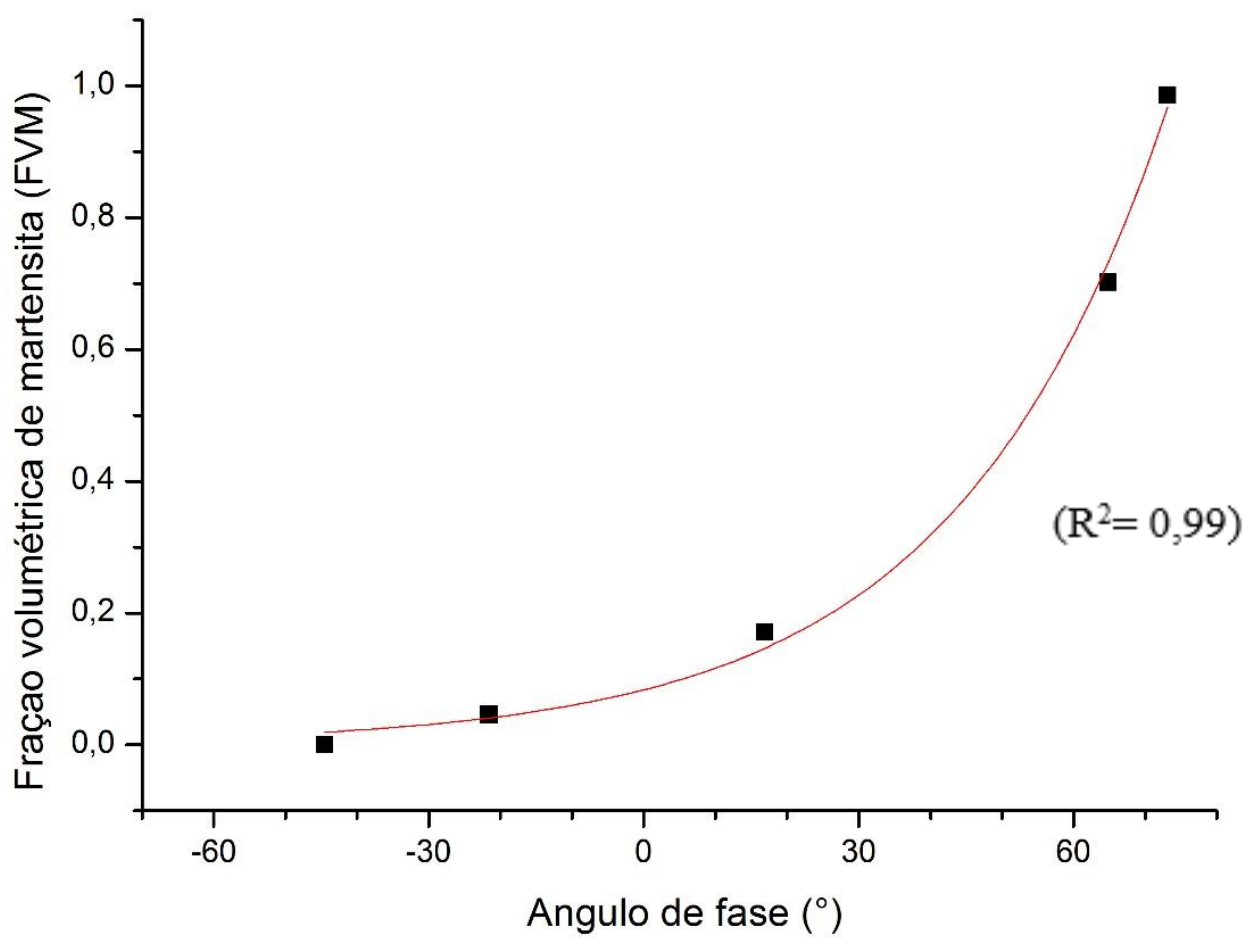

Figura 4. Relação entre a fração volumétrica de martensita e o ângulo de fase.

$$
F V M=0,08 \cdot \exp (-A F /-29,69)+9,28 \cdot 10^{-4}
$$

Com a calibração realizada a partir da equação anterior, foram realizadas medidas de ângulos de fases em algumas amostras, e a fração de martensita obtida de maneira não destrutiva. A Figura 5 mostra os resultados de correntes parasitas sobrepostos aos mostrados para o VSM na Figura 3, até a deformação de 1,0. É possível observar que ambos revelam uma curva sigmoidal, que é o comportamento da transformação martensítica observada nestes aços, como relatado em [10]. Além disso, os resultados de correntes parasitas estão muito próximos aos encontrados pelo VSM, denotando que a curva de calibração estabelecida neste trabalho foi efetiva para estimar a fração de martensita a partir do ensaio de correntes parasitas. 


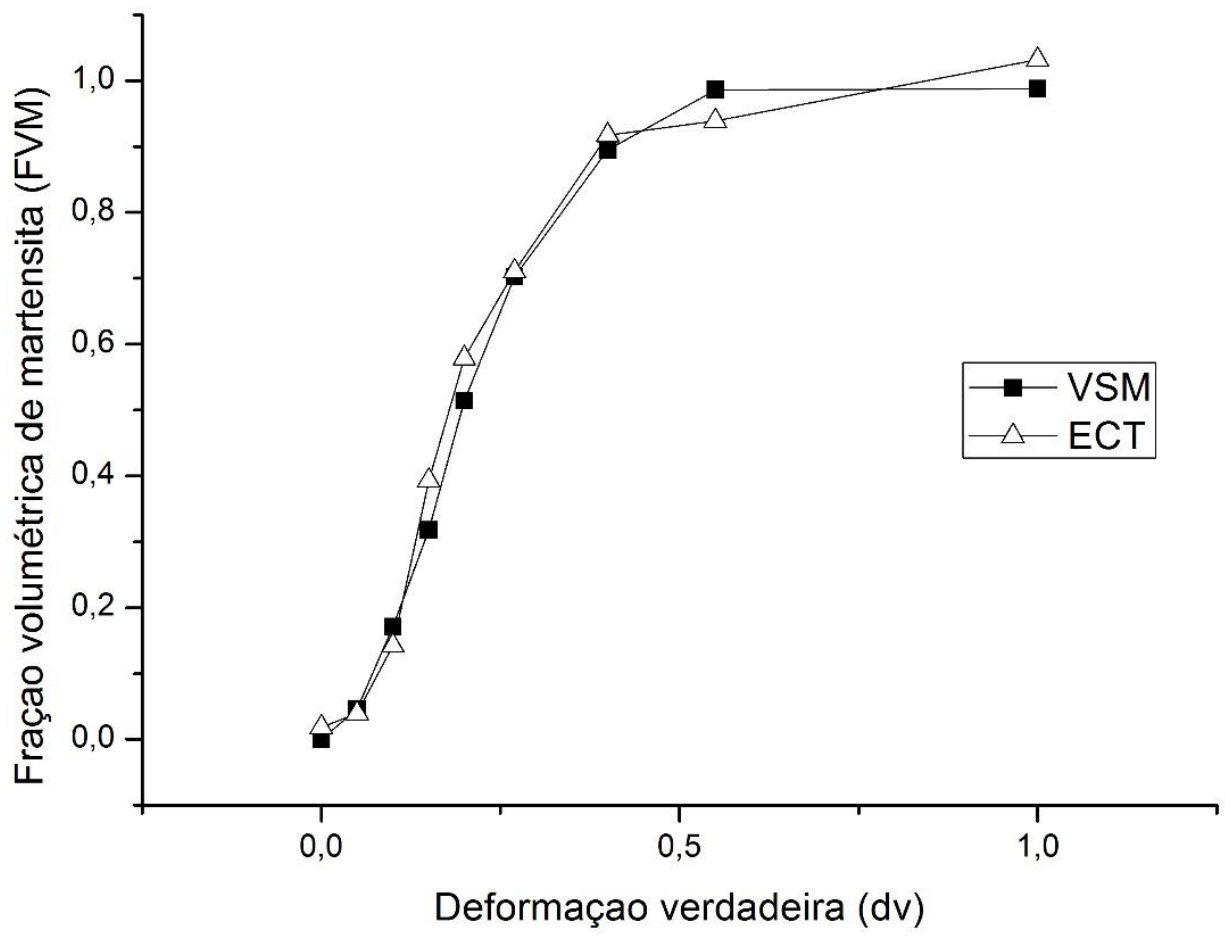

Figura 5. Comparação da fração volumétrica de martensita obtida por VSM e ECT.

\section{CONCLUSÃo}

Observa-se que a transformação martensítica no aço AISI 321 ocorre predominantemente entre os estágios de 0,1 e 0,5 em deformação verdadeira. Com os dados da fração volumétrica de martensita, um ajuste exponencial apresentou-se como boa opção para servir como uma curva de calibração para o ensaio de correntes parasitas, já que a quantificação de fase por ECT apresentou resultados muito próximos àqueles obtidos por VSM.

\section{Agradecimentos}

Os autores agradecem ao apoio financeiro da Capes e do CNPQ.

\section{REFERÊNCIAS}

1 Dash J, Otte HM. The martensite transformation in stainless steel. Acta Metallurgica. 1963; 11(10): 1169-1178.

2 Mangonon PL, Thomas G. The martensite phases in 304 stainless steel. Metall. Trans.; 1970;1:1577-1586.

3 Smaga M, Walther F, Eifler D. Deformation-induced martensitic transformation in metastable austenitic steels. Materials Science and Engineering A. 2008; 483:394-397. 
4 Talonen J, Hänninen H. Formation of Shear Bands and Strain-Induced Martensite During Plastic Deformation of Metastable Austenitic Stainless Steels. Acta Materialia. 2007; 55 (18): 6108 - 6118.

5 Tavares SSM, Neto JM, Silva MR, Vasconcelos IF, Abreu HFG. Magnetic properties and $\alpha$ martensite quantification in an AISI 301LN stainless steel deformed by cold rolling. Materials Characterization. 2008; 59: 901-904,

6 Talonen J, Aspegren P, Hänninen H. Comparison of different methods for measuring strain induced' $\alpha$ - martensite content in austenitic steels. Mater. Sci. Tech. 2004; 20: $1506-1512$.

7 Kisko A, Misra RDK, Talonen J, Karjalainen LP. The influence of grain size on the straininduced martensite formation in tensile straining of an austenitic $15 \mathrm{Cr}-9 \mathrm{Mn}-\mathrm{Ni}-\mathrm{Cu}$ stainless steel. Materials Science and Engineering: A. 2013; 578:408-416.

8 Hedayati A, Najafizadeh A, Kermanpur A, Forouzan F. The effect of cold rolling regime on microstructure and mechanical properties of AISI 304L stainless steel. Journal of Materials Processing Technology. 2010; 210: 1017-1022.

9 Khan SH, Ali F, Khan AN, et al. Eddy current detection of changes in stainless steel after cold reduction. Computational Materials Science. 2008; 43: 623628.

10 Surkiliabad R, Hedayati A, Alam AS. Monitoring of martensitic transformation in coldrolled 304L austenitic stainless steel by eddy current method. E-Journal of Nondestructive Testing. 2009; 2(10): 312-323.

11 Tavares SSM, Pardal JM, da Silva MR, de Oliveira CAS. Martensitic Transformation Induced by Cold Deformation of Lean Duplex Stainless Steel Uns S32304. Materials Research. 2014; 17(2): 381-385.

12 Tavares MT. Avaliação da Transformação Martensítica por Deformação a Frio no Aço Inoxidável Austenítico AISI 321 Contendo Diversos Teores de Carbono. Dissertação de M. Sc., PGMEC/ UFF, Niterói, RJ, Brasil, 2014.

13 Shirdel M, Mirzadeh H, Parsa MH. Estimation of the kinetics of martensitic transformation in austenitic stainless steels by conventional and novel approaches. Materials Science and Engineering: A. 2015; 624: 256-260.

14 Hausild P, Davydov V, Drahokoupil J, Landa M, Pilvin P. Characterization of straininduced martensitic transformation in a metastable austenitic stainless steel. Materials and Design. 2010; 31:1821-1827. 